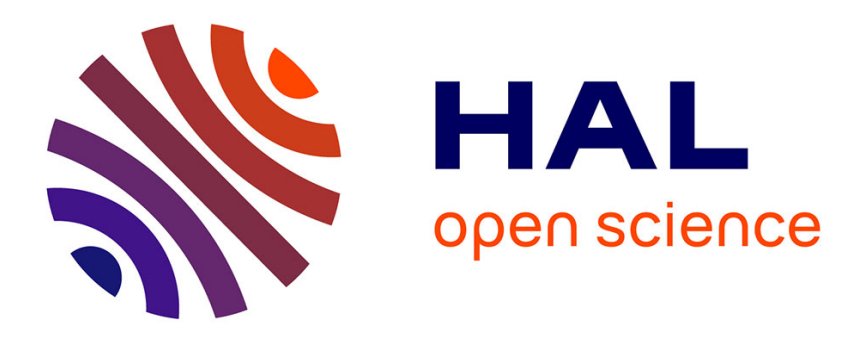

\title{
Synchronizability of nonidentical weakly dissipative systems
}

Irene Sendina-Nadal, Christophe Letellier

\section{To cite this version:}

Irene Sendina-Nadal, Christophe Letellier. Synchronizability of nonidentical weakly dissipative systems. Chaos: An Interdisciplinary Journal of Nonlinear Science, 2017, 27 (10), 10.1063/1.5005840 . hal-01672078

\section{HAL Id: hal-01672078}

https://hal-normandie-univ.archives-ouvertes.fr/hal-01672078

Submitted on 23 Dec 2017

HAL is a multi-disciplinary open access archive for the deposit and dissemination of scientific research documents, whether they are published or not. The documents may come from teaching and research institutions in France or abroad, or from public or private research centers.
L'archive ouverte pluridisciplinaire HAL, est destinée au dépôt et à la diffusion de documents scientifiques de niveau recherche, publiés ou non, émanant des établissements d'enseignement et de recherche français ou étrangers, des laboratoires publics ou privés. 


\section{Synchronizability of nonidentical weakly dissipative systems}

Irene Sendiña-Nadal, and Christophe Letellier

Citation: Chaos 27, 103118 (2017); doi: 10.1063/1.5005840

View online: http://dx.doi.org/10.1063/1.5005840

View Table of Contents: http://aip.scitation.org/toc/cha/27/10

Published by the American Institute of Physics 


\title{
Synchronizability of nonidentical weakly dissipative systems
}

\author{
Irene Sendiña-Nadal ${ }^{1,2}$ and Christophe Letellier ${ }^{3}$ \\ ${ }^{1}$ Complex Systems Group \& GISC, Universidad Rey Juan Carlos, 28933 Móstoles, Madrid, Spain \\ ${ }^{2}$ Center for Biomedical Technology, Universidad Politécnica de Madrid, 28223 Pozuelo de Alarcón, \\ Madrid, Spain \\ ${ }^{3}$ CORIA-UMR 6614, Normandie Université, Campus Universitaire du Madrillet, F-76800 Saint-Etienne \\ du Rouvray, France
}

(Received 23 January 2017; accepted 19 September 2017; published online 17 October 2017)

\begin{abstract}
Synchronization is a very generic process commonly observed in a large variety of dynamical systems which, however, has been rarely addressed in systems with low dissipation. Using the Rössler, the Lorenz 84, and the Sprott A systems as paradigmatic examples of strongly, weakly, and non-dissipative chaotic systems, respectively, we show that a parameter or frequency mismatch between two coupled such systems does not affect the synchronizability and the underlying structure of the joint attractor in the same way. By computing the Shannon entropy associated with the corresponding recurrence plots, we were able to characterize how two coupled nonidentical chaotic oscillators organize their dynamics in different dissipation regimes. While for strongly dissipative systems, the resulting dynamics exhibits a Shannon entropy value compatible with the one having an average parameter mismatch, for weak dissipation synchronization dynamics corresponds to a more complex behavior with higher values of the Shannon entropy. In comparison, conservative dynamics leads to a less rich picture, providing either similar chaotic dynamics or oversimplified periodic ones. Published by AIP Publishing. https://doi.org/10.1063/1.5005840
\end{abstract}

Weakly dissipative systems, typically found in celestial mechanics, fluid mechanics, meteorology, mechanics, ecology, etc., have solutions that visit a non-zero volume of the state space in contrast with those being strongly dissipative. As a consequence, the characterization of their chaotic attractor is much more complicated. When synchronization is considered, it is of great interest to consider if strongly or weakly dissipative systems behave similarly or not. Here, we compared, by using the Shannon entropy, the way nonidentical systems presenting different dissipation rates organize into a collective dynamics and found that low dissipation favors an increase of the Shannon entropy of the interacting systems while high dissipation gives rise to a dynamics whose complexity is in between of the coupled ones.

\section{INTRODUCTION}

The analysis of the chaotic behavior produced by a dynamical system can be difficult to perform in the context of a weak energy dissipation scenario. In such a case, there is no rapid relaxation of the trajectories to the central manifold and the attractor visits a significant volume of the state space. Consequently, the chaotic attractor produced by a weakly dissipative system features a "thick" Poincaré section, contrary to what is observed when there is strong dissipation for which the thickness of such a section can be neglected, as well exemplified by the Rössler attractor. ${ }^{1}$ In this latter case, attractors are commonly characterized by a branched manifold (a template) revealing their most relevant topological properties. ${ }^{2-4}$ For weakly dissipative systems instead, uncovering a branched manifold from the data is not an easy task and an analysis based on symbolic dynamics can be astonishingly cumbersome due to the difficulty to define a good partition of the attractor. ${ }^{5}$ Since chaotic attractors are structured around a population of unstable periodic orbits, ${ }^{6,7}$ its extraction is a necessary step for their characterization. While it is straightforward to accomplish such a task for strongly dissipative systems using closed return maps, ${ }^{8}$ unveiling the structure of chaotic attractors produced by weakly dissipative systems remains a rather challenging issue to achieve.

In spite of the interesting paradigm they offer in various fields, ${ }^{9-12}$ the study of how two weakly dissipative systems synchronize seems to be an elusive topic as confirmed, to the best of our knowledge, by the lack of clear results in the literature. The case of synchronizing conservative systems has also been rarely investigated. ${ }^{13-15}$ The reason behind such a reduced amount of works devoted to weakly dissipative systems is the coexistence of many attractors in the state space $^{16,17}$ and the difficulty to characterize them as previously discussed. We thus investigate the synchronizability of systems having low dissipation rate and address how it differs from the very well-known behavior exhibited by strongly dissipative ones. The case of conservative systems will be also discussed.

The subsequent part of this paper is organized as follows. Section II is devoted to the characterization of the three systems considered: (i) the strongly dissipative Rössler system, (ii) the weakly dissipative Lorenz 84 system, and (iii) the conservative Sprott A system. Section III addresses how the resulting collective dynamics is affected by a parameter or frequency mismatch between two coupled oscillators. Section IV gives some conclusions to the paper. 


\section{DYNAMICS CHARACTERIZATION}

Commonly, in order to characterize chaotic attractors produced by strongly dissipative systems, a first-return map is preferred over a Poincaré section because its structure is very sensitive to the population of unstable periodic orbits. ${ }^{18,19}$ However, in the case of toroidal and/or weakly dissipative dynamics, the structure of the thick Poincaré section prevents the computation of a non-self-intersecting firstreturn map. These self-intersections are the source of the difficulties encountered for describing the structure of these types of attractors. In fact, periodic orbits cannot be easily identified and it is rather challenging to propose a branched manifold describing the main topological properties of the chaotic attractor (see Ref. 20 for a first attempt to characterize the topology of a weakly dissipative dynamics). In the following, first-return maps to a Poincaré section will be used to unfold the dynamics underlying strongly dissipative systems, while Poincaré sections will do for the weakly dissipative and conservative systems. In addition, we will refer to a period- $p$ limit cycle, when the trajectory crosses $p$-times the Poincaré section before repeating itself.

Since an accurate topological description of a weakly dissipative dynamics is difficult to carry out, we opted instead to use the Shannon entropy computed from recurrence plots. ${ }^{21,22}$ This measure increases with the development of the population of unstable periodic orbits and is more reliable when computed from a Poincaré section or a first-return map than from a continuous time series. ${ }^{21}$ Let us consider the discrete time series $\left\{\boldsymbol{u}_{i}\right\}_{i=1}^{N}$, where the $N$ points $\boldsymbol{u}_{i}$ come either from a Poincaré section $\boldsymbol{u}_{i}=\left(x_{i}, y_{i}\right)$ or a firstreturn map $\boldsymbol{u}_{i}=\left(y_{i}, y_{i+1}\right)$. Two points $\boldsymbol{u}_{i}$ and $\boldsymbol{u}_{j}$ are considered as being recurrent if

$$
\left\|\boldsymbol{u}_{i}-\boldsymbol{u}_{j}\right\|=\sqrt{\left(x_{j}-x_{i}\right)^{2}+\left(y_{j}-y_{i}\right)^{2}}<\epsilon,
$$

with

$$
\epsilon=\frac{1}{10} \sqrt{\left(X_{\max }-X_{\min }\right)^{2}+\left(Y_{\max }-Y_{\min }\right)^{2}},
$$

where $X_{\min , \max }$ and $Y_{\min , \max }$ values correspond to the largest domain visited in the state space of a given system. By using the condition given by Eq. (1), we construct the so-called recurrence plot $R_{i j}$ as an $N \times N$ square matrix

$$
R_{i j}=\theta\left(\epsilon-\left\|\boldsymbol{u}_{i}-\boldsymbol{u}_{j}\right\|\right),
$$

where $\theta\left(\boldsymbol{u}_{i}\right)$ is the Heaviside function. According to Refs. 21 and 23, the Shannon entropy is defined as

$$
S_{h}=-\sum_{n=1}^{M} P_{n} \log \left(P_{n}\right),
$$

where $M$ is the maximum length among all the segments parallel to the main diagonal of $R_{i j}$ with non-recurrent points, and $P_{n}$ is the number of segments with $n$ non-recurrent points divided by the total number of recurrent points in the considered recurrence plot. ${ }^{23}$

\section{A. The strongly dissipative Rössler system}

We consider the Rössler system ${ }^{1}$ as a model of a strongly dissipative system

$$
\left\{\begin{array}{l}
\dot{x}=\omega[-y-z] \\
\dot{y}=\omega[x+a y] \\
\dot{z}=\omega[b+z(x-c)],
\end{array}\right.
$$

which produces a chaotic attractor as the one shown in Fig. 1(a). A suitable Poincaré section is defined as

$$
\mathcal{P}_{\text {ros }} \equiv\left\{\left(y_{n}, z_{n}\right) \in \mathbb{R}^{2} \mid x_{n}=x_{\mathrm{p}}, \dot{x}_{n}>0\right\},
$$

where $x_{\mathrm{p}}=\frac{c-\sqrt{c^{2}-4 a b}}{2}$ is the $x$-value of the inner singular point of the Rössler system. As previously discussed, chaotic attractors produced by strongly dissipative systems are better characterized by a first-return map to a Poincaré section than by a Poincaré section. While the Poincaré section plotted in Fig. 1(b) looks like a simple arc from which no useful information can be easily extracted, the first-return map usually exhibits monotonic branches separated by critical points-extremaas shown in Figs. 2(b)-2(d). A first-return map with $k$ critical points is said to be a $k$-modal map, ${ }^{18,19} \mathrm{Fig}$. 2(b) being an example of a three-modal map with four branches (labelled from 0 to 3 next to each branch) and three critical points.

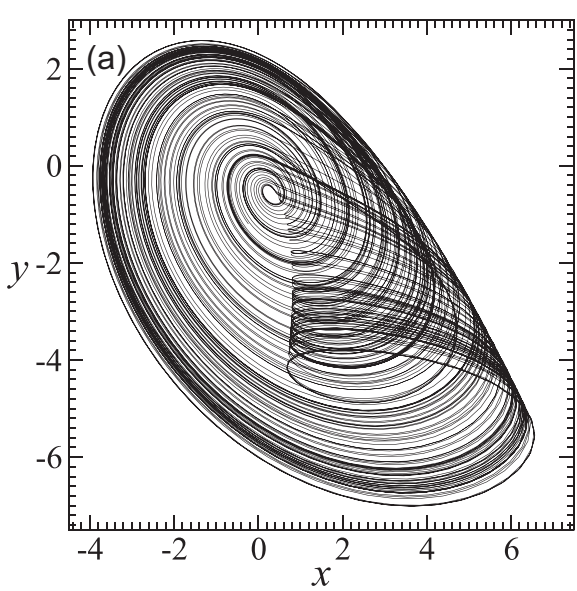

State portrait

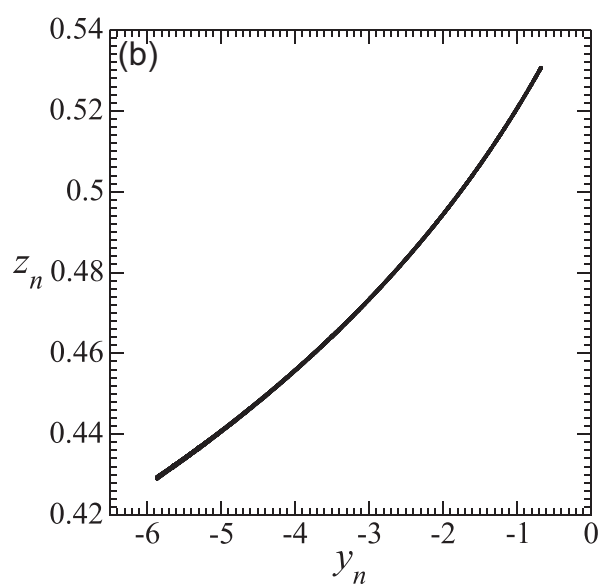

Poincaré section $\mathcal{P}_{\text {Ros }}$
FIG. 1. Chaotic attractor produced by the Rössler system. (a) Chaotic attractor. (b) Poincaré section. Parameter values: $a=0.520, b=2, c=4$, and $\omega=5$. 

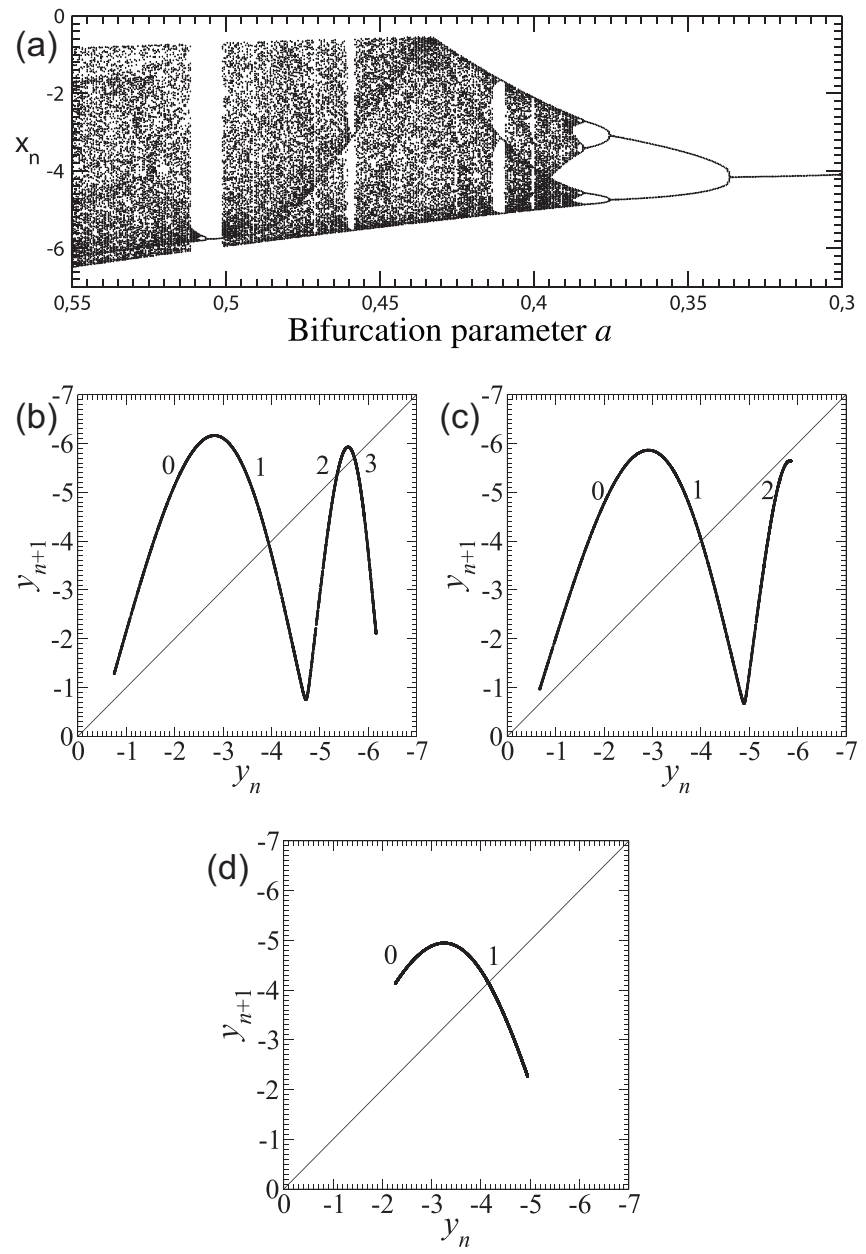

FIG. 2. Bifurcation diagram (a) and first-return maps to a Poincaré section [(b) $a=0.520, S_{h}=2.50$, (c) $a=0.492, S_{h}=2.42$ and (d) $a=0.395$, $\left.S_{h}=1.20\right]$ of three different chaotic attractors produced by the Rössler system. Each branch in the first-return maps is labelled with an integer. Other parameter values: $b=2, c=4$, and $\omega=5$.0.

The corresponding Jacobian matrix of the system (5) is

$$
\mathcal{J}=\omega\left[\begin{array}{ccc}
0 & -1 & -1 \\
1 & +a & 0 \\
z & 0 & x-c
\end{array}\right] .
$$

The time averaged trace $\overline{\mathrm{Tr}}=\overline{\omega(a \bar{c}+x)^{38}}$ accounts for the dissipation rate and it ranges between -13.5 and -15.9 in the interval of inspected $a$ values, $a=[0.395,0.520]$, with a mean value of $-15.0 \pm 1.0$. The dissipation is, therefore, not significantly affected by the change in the parameter $a$ used in this study.

Parameter $\omega$ is introduced to renormalize the time while leaving unchanged the bifurcation diagram versus parameter $a$. For $a=0.520$, the dynamics is non-phase-coherent as shown by the distribution of the oscillation periods whose mean value is $1.54 \pm 0.27 \mathrm{~s}$ (Fig. 3). Note that the non-phasecoherence is here due to the presence of two rather welldefined peaks. When parameter $a$ is decreased, the first-return map becomes far less developed [Figs. 2(c) and 2(d)] and the dynamics becomes phase-coherent when only branches 0 and 1 are observed in the first-return map. ${ }^{19}$ The values of the Shannon entropy are also reported, showing that the more developed the dynamics is, the greater the entropy is.

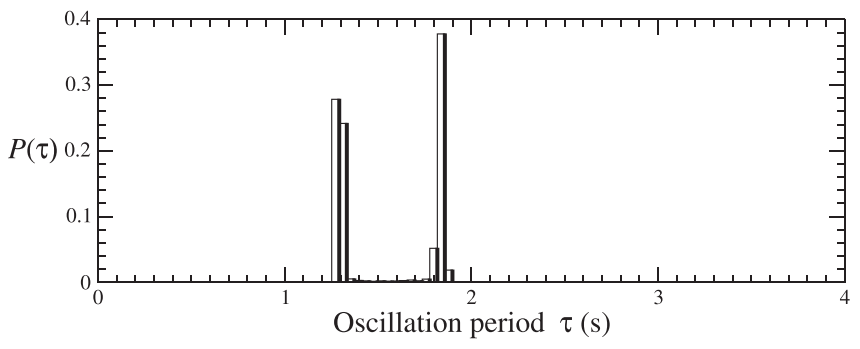

FIG. 3. Distribution of the oscillation periods for a trajectory within the attractor produced by the Rössler system. Mean oscillation period: $\bar{\tau}=$ $1.54 \pm 0.27 \mathrm{~s}$. Parameter values: $a=0.520, b=2, c=4$, and $\omega=5.0$.

\section{B. The weakly dissipative Lorenz 84 system}

In 1984, Edward Lorenz proposed the simple model

$$
\left\{\begin{array}{l}
\dot{x}=-y^{2}-z^{2}-a x+a F \\
\dot{y}=x y-b x z-y+G \\
\dot{z}=b x y+x z-z
\end{array}\right.
$$

for the global atmospheric circulation. ${ }^{24}$ Typical parameter values are $a=0.28, b=4, F=8$, and $G=1$, which produce the chaotic attractor shown in Fig. 4. These values will be used throughout this work unless otherwise specified. The structure of this attractor is rather complicated due to its toroidal structure as clearly shown in the $y-z$ plane projection.

The trace of the Jacobian matrix

$$
\mathcal{J}=\left[\begin{array}{ccc}
-a & -2 y & -2 z \\
y-b z & x-1 & -b x \\
b y+z & b x & x-1
\end{array}\right]
$$

is not constant and equal to

$$
\operatorname{Tr}(\mathcal{J})=-a+2(x-1) .
$$

Its mean value is equal to $-0.25 \pm 0.08$ in the interval $a$ $=[0.235,0.28]-60$ times smaller than that of the Rössler system-confirming the low dissipation rate of the Lorenz 84 system with respect to the Rössler in the parameter space

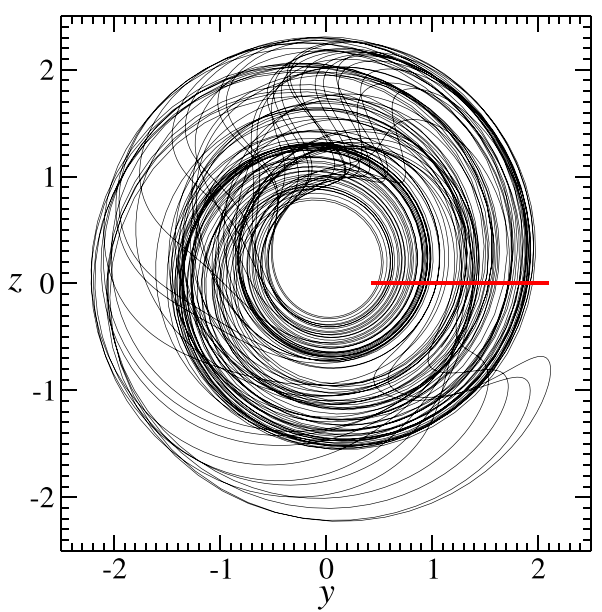

FIG. 4. Chaotic attractor produced by the Lorenz 84 system (8). The $y-z$ plane projection evidences the main rotation driving the dynamics of the Lorenz 84 system. The Poincaré plane is drawn as a red line. Parameter values are $a=0.28, b=4, F=8$, and $G=1$. 


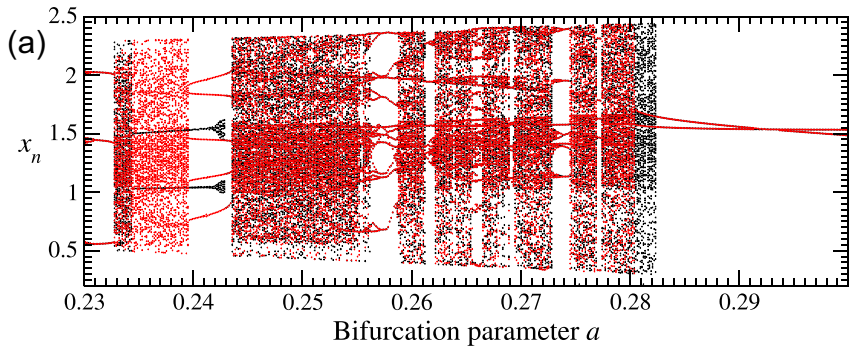

(b)
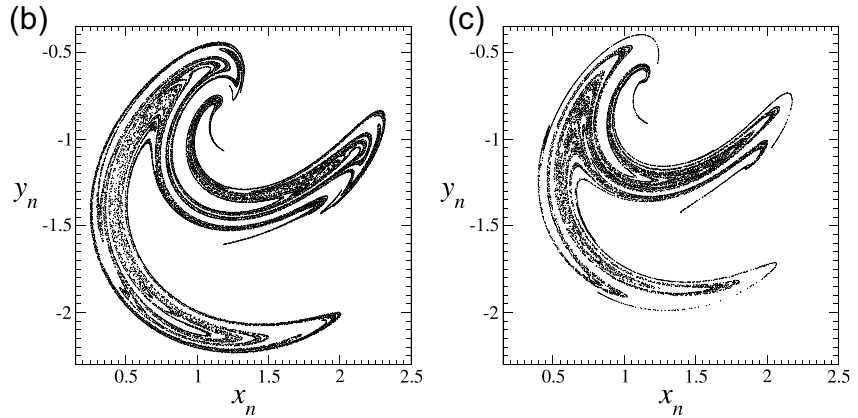

FIG. 5. Bifurcation diagram of the Lorenz 84 system (8) and Poincaré sections of two of the chaotic attractors it produces. The bifurcation diagram is computed by increasing (black) and decreasing (red) the $a$-value. In spite of the apparent similarities between the two sections, the volume visited in $a=0.28, S_{h}=1.52$ (b) $\left(\mathcal{V}_{0.280}=13.1 \%\right)$ is nearly the double in $a=0.235$, $S_{h}=1.21$ (c) $\left(\mathcal{V}_{0.235}=6.6 \%\right)$. The Poincare plane used to compute the Poincaré section is given by Eq. (11) and drawn as a red line in Fig. 4. The rest of parameter values as in Fig. 4.

explored. This is further evidenced by the thickness of the Poincaré section

$$
\mathcal{P}_{\text {Lor }} \equiv\left\{\left(x_{n}, y_{n}\right) \in \mathbb{R}^{2} \mid z_{n}=0, \dot{z}_{n}<0\right\},
$$

which reveals a rich layered structure as exemplified in Figs. 5(b) and 5(c) for two different $a$-values. Two aspects indicate that these two regimes have distinct properties: (i) the visited volume $\mathcal{V}$ of the state space ${ }^{39}$ for $a=0.28$ [Fig. 5(b)] is about the double of what it is for $a=0.235$ [Fig. 5(c)] and (ii) the layered structure of the two Poincaré sections are qualitatively different [compare the middle top of Fig. 5(b) with the top of Fig. 5(c)]. This change is quantified by the corresponding Shannon entropy values $S_{h}=1.52$ and $S_{h}=1.21$, respectively. Therefore, the two populations of unstable periodic orbits are not alike, and the chaotic attractor in Fig. 5(b) is more developed than the one shown in Fig. 5(c).

The bifurcation diagram versus the parameter $a$ [Fig. 5 (a)] reveals a very rich collection of bifurcations. ${ }^{25-28}$ The co-existence of at least two attractors is shown by computing the bifurcation diagram by increasing (black) and decreasing (red) the parameter $a$. For instance, when $a$ is increased, a period-2 limit cycle [whose upper segment is shown in the blowup of Fig. 6(a)] is destabilized through a Hopf bifurcation at $a \approx 0.2416$ leading to a quasi-periodic regime [Fig. 6(c)] with its characteristic annular Poincare section (not shown). The trajectory thus settles down onto an invariant torus. This quasi-periodic regime is further destabilized through a boundary crisis with a period- 8 unstable orbit. Once the torus collides with the period- 8 orbit, the trajectory slowly approaches the domain where the invariant torus was
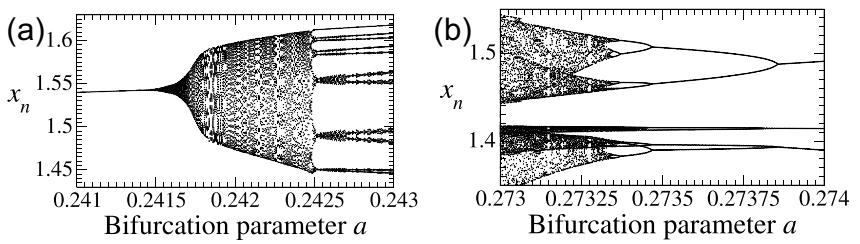

(c)
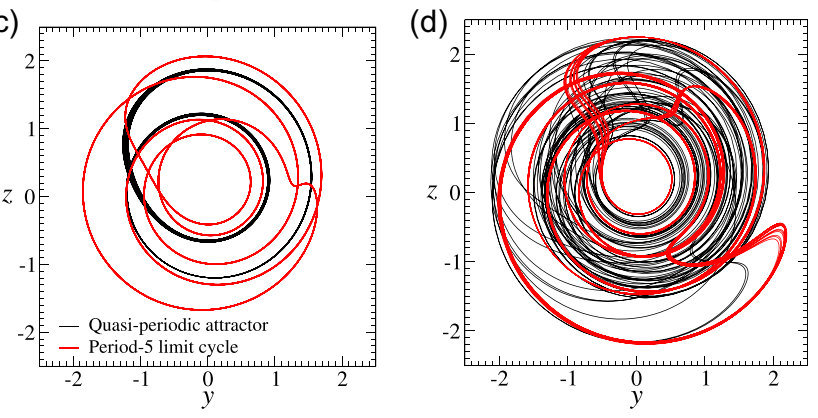

FIG. 6. (a) and (b) Blowups of the bifurcation diagram of the Lorenz 84 system shown in Fig. 5(a) to evidence its rich structure. (a) A Hopf bifurcation of a period-2 limit cycle leads to (c) a quasi-periodic behavior (black) coexisting with a period-5 limit cycle (red) in the state space at $a=0.2417$. (b) A period-doubling (PD) cascade issued ( $a$ decreasing) from a period-7 limit cycle leads to (d) a chaotic attractor (red) coexisting with a more developed one (black) for $a=0.273$.

before the crisis, and then spirals very slowly toward the now stable period- 8 orbit. All these attractors (from the period-2 limit cycle to the quasi-periodic regime becoming a period-8 limit cycle) coexist in the state space with a period5 limit cycle [Fig. 6(c)]. A second example of bifurcation [Fig. 5(a), red] is a period-doubling cascade when $a$ is decreased from 0.274 [Fig. 6(b)]. It starts with a period-7 limit cycle [Fig. 6(d), red] coexisting with a chaotic attractor [Fig. 6(d), black].

The state space of the Lorenz 84 system is definitely not as simple as commonly observed in strongly dissipative ones. Nevertheless, as shown in Figs. 4, 6(c), and 6(d) or by the bifurcation diagram in Fig. 5(a), the visited domain of the state space is bounded and it is not significantly affected by the multistability.

The chaotic attractor produced by the Lorenz 84 system is non-phase-coherent as shown by the probability distribution $P(\tau)$ of the oscillation periods $\tau$ (Fig. 7). The mean period is $\bar{\tau}=1.54 \pm 0.77 \mathrm{~s}$ as for the Rössler system (Fig. 3) with a peak at a typical period $\tau_{\mathrm{t}}=0.85 \mathrm{~s}$ and oscillation periods extending four times longer. The non-phase-coherence is thus due to a large flat distribution and not to two rather well-defined oscillation periods as in the Rössler system. The time scales of the two systems are chosen to be of

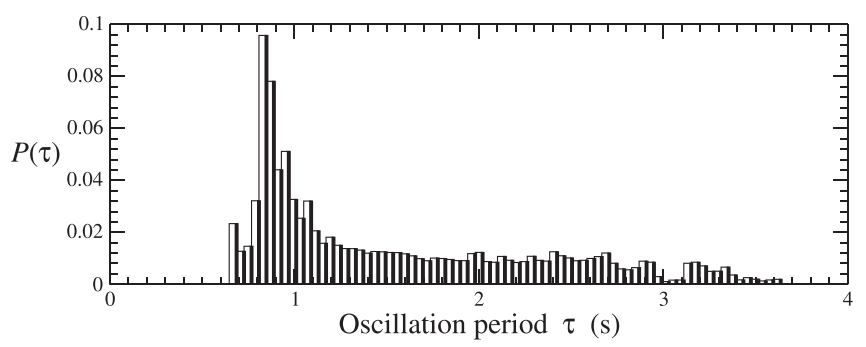

FIG. 7. Distribution of the oscillation periods $\tau$ described by a trajectory within the attractor produced by the Lorenz 84 system. $\bar{\tau}=1.54 \pm 0.77 \mathrm{~s}$. Parameter values as in Fig. 4. 
the same order because this is relevant for comparing their dissipation rates (which accounts for the volume contraction per unit of time). Indeed, the dissipation rate is associated with the strength of the squeezing mechanism occurring after the stretching-and-folding mechanism responsible for the chaotic dynamics. ${ }^{2}$ The strength of the squeezing mechanism in chaotic attractors affects directly the Poincaré section and first-return map thickness.

\section{The conservative Sprott A system}

Among the collection of simple chaotic systems found by Sprott, ${ }^{29}$ the Sprott A system

$$
\left\{\begin{array}{l}
\dot{x}=\omega y \\
\dot{y}=\omega(-x+y z) \\
\dot{z}=\omega\left(a-y^{2}\right)
\end{array}\right.
$$

has the particularity of being conservative as evidenced by its Jacobian matrix

$$
\mathcal{J}=\omega\left[\begin{array}{ccc}
0 & 1 & 0 \\
-1 & z & y \\
0 & -2 y & 0
\end{array}\right]
$$

whose trace is such that

$$
\overline{\operatorname{Tr}}=\lim _{T \rightarrow \infty} \omega \int_{t=0}^{T} z \mathrm{~d} t=0 .
$$

Therefore, there is no longer an attractor and the nature of the solution strongly depends on the initial conditions as shown by the Poincaré section

$$
\mathcal{P}_{\text {Spr }} \equiv\left\{\left(x_{n}, y_{n}\right) \in \mathbb{R}^{2} \mid z_{n}=0\right\},
$$

depicted in Figs. 8(b) and 8(c).
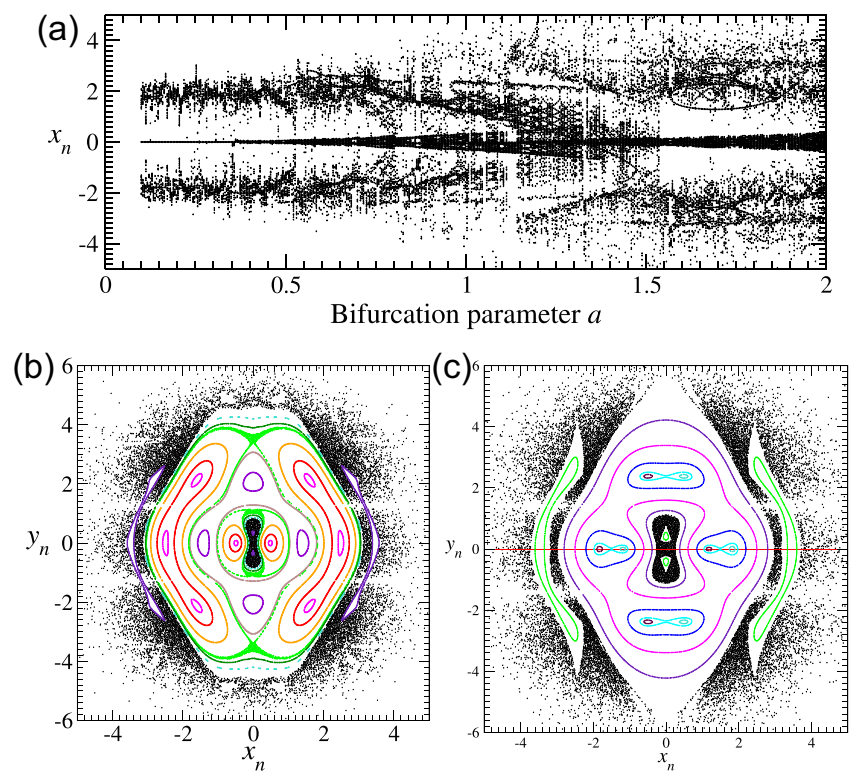

FIG. 8. (a) Bifurcation diagram of the Sprott A system versus parameter $a$, and (b) and (c) Poincaré sections $(z=0)$ of the solutions produced for two different values of parameter $a$ [(b) $a=1.6, S_{h}=1.97$ and (c) $a=2.0$, $\left.S_{h}=2.04\right]$. Other parameter values: $\omega=3.41$. See the main text for the values of the initial conditions used for each $a$-value.

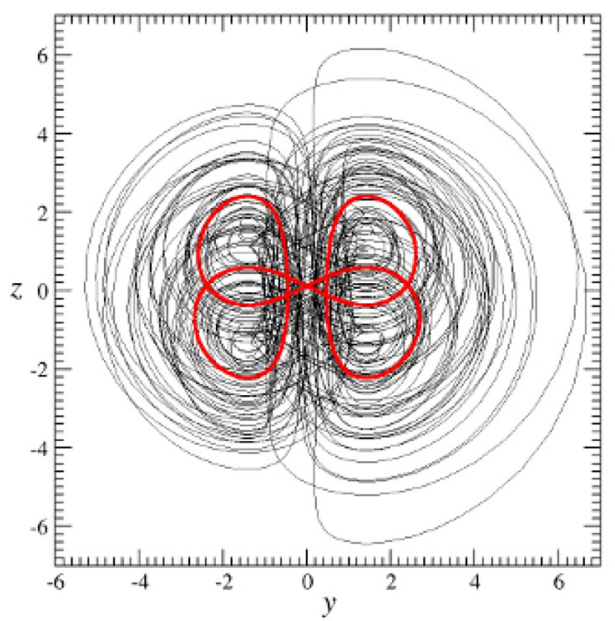

FIG. 9. Co-existing solutions produced by the Sprott system. The initial conditions for the period- 4 limit cycle are chosen in the center of one of the four (blue) islands shown in Fig. 8(c). Parameter values as in Fig. 8(c).

For $a=2.0$ and $\omega=3.41$, a chaotic sea is observed (black dots in Fig. 8), for instance, from the initial conditions

$$
\mid \begin{aligned}
& x_{0}=2.5 \omega \\
& y_{0}=3.1 \omega \\
& z_{0}=0 \omega
\end{aligned}
$$

This so-called chaotic sea surrounds quasi-periodic islands among which the central one is structured around a symmetric period-4 limit cycle (red trajectory in Fig. 9) for all the used $a$-values. We adjusted $\omega=3.41$ to have a mean oscillation period nearly equal to those observed for the two previous systems. Choosing the initial conditions as in (16) allows to leave the dynamics invariant under any change in $\omega$ and to ensure that we are still in the chaotic sea. The Sprott A system combines many different coexisting solutions in the state space with a distribution of the oscillation periods characterized by two peaks (see Fig. 10). The bifurcation diagram [Fig. 8(a)] versus parameter $a$ (the initial conditions being the same for each $a$-value) shows how the chaotic sea evolves: its size increases when $a$ is increased.

We have thus retained three systems whose dissipation rates can be significantly different. A related property is that the Rössler system rarely exhibits multistability, while the two other produce solutions that depend on the initial conditions but, as we will see, such a dependency is not relevant to our results.

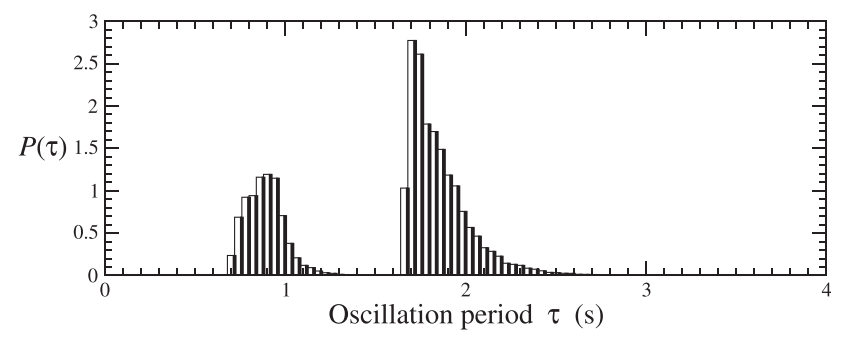

FIG. 10. Distribution of the oscillation periods $\tau$ for a trajectory within the attractor produced by the Sprott A system. Mean oscillation period: $\bar{\tau}=$ $1.55 \pm 0.46$ s. Parameter values as in Fig. 8 . 


\section{SYNCHRONIZABILITY OF TWO COUPLED SYSTEMS}

Let us consider the equation

$$
\dot{\boldsymbol{x}}_{1,2}=\boldsymbol{f}\left(\boldsymbol{x}_{1,2}\right)-\rho\left[\boldsymbol{h}\left(\boldsymbol{x}_{1,2}\right)-\boldsymbol{h}\left(\boldsymbol{x}_{2,1}\right)\right],
$$

governing the time evolution of two coupled oscillators, where $\boldsymbol{f}\left(\boldsymbol{x}_{i}\right)$ stands for the vector flow and $\boldsymbol{h}\left(\boldsymbol{x}_{i}\right)$ and $\rho$ are the coupling function and global coupling parameter, respectively. ${ }^{30,31}$

According to the master stability function approach for assessing the synchronizability of identical systems, ${ }^{32}$ complete synchronization of Lorenz 84 systems is realized when coupled through variable $z$, that is, $\boldsymbol{h}=(0,0, z)$ in Eq. (17). A similar situation is obtained with Rössler systems when they are coupled through the variable $x$ and the Sprott A systems via variable $y$. In these three cases, the synchronous state $\boldsymbol{x}_{1}=\boldsymbol{x}_{2}$ is stable within an interval of the coupling parameter. ${ }^{33,34}$

When the coupled systems are nonidentical, the complete synchronization is no longer an invariant manifold of the dynamics and the stability of the almost synchronous solution is reduced. ${ }^{35,36}$ In order to check how a parameter or frequency mismatch affects the synchronization and the resulting dynamics, we introduced a mismatch in the $a_{2}$ parameter of the second oscillator while keeping fixed the $a=a_{1}$ value of the first one. A standard marker for the quality of the synchronization is the normalized average synchronization error

$$
e=\lim _{T \rightarrow \infty} \frac{1}{T} \int_{0}^{T} \frac{1}{D}\left\|\boldsymbol{x}_{2}(t)-\boldsymbol{x}_{1}(t)\right\| d t
$$

where \|\| stands for the Euclidean norm and $D=$ $\max \left(\left\|\boldsymbol{x}_{2}(t)-\boldsymbol{x}_{1}(t)\right\|\right)$ is the diameter of the attractor. Together with the synchronization error, we will use the Shannon entropy to characterize the resulting dynamics from the interaction of the two oscillators as a function of the dissipation rate.

\section{A. Mismatch in one parameter value}

We computed the relative Shannon entropy $\tilde{S}_{h}$ and the normalized synchronization error $e$, while varying the coupling strength $\rho$ and the ratio $\frac{\Delta a}{a_{1}}=1-\frac{a_{2}}{a_{1}}$ for the three systems. The Shannon entropy $\tilde{S}_{h}$ is normalized to the reference value of the Shannon entropy $S_{h}$ of the first oscillator when it is uncoupled. In Fig. 11, we report $\tilde{S}_{h}$ (left column) and the normalized synchronization error $e$ (right column) for the three systems. For the strongly dissipative case, the Shannon entropy tends to decrease when $\rho$ increases [Fig. 11(a)]. On the other hand, there is a threshold value $\left(\rho_{x} \approx 4\right)$ beyond which the trajectory is ejected to infinity. In this "bifurcation" diagram, when $1.5<\rho_{x}<4$, there are some regions where the Shannon entropy and the synchronization are nearly zero, suggesting the emergence of a common limit cycle. The parameter mismatch, therefore, plays the role of a bifurcation parameter. For instance, when $\rho_{x}=2.5$ and $\frac{\Delta a}{a_{1}}=5.4 \%$ ( $a_{1}=0.520$ and $a_{2}=0.492$ ), the structure of the attractor is already modified [Fig. 12(a)] and the corresponding first-
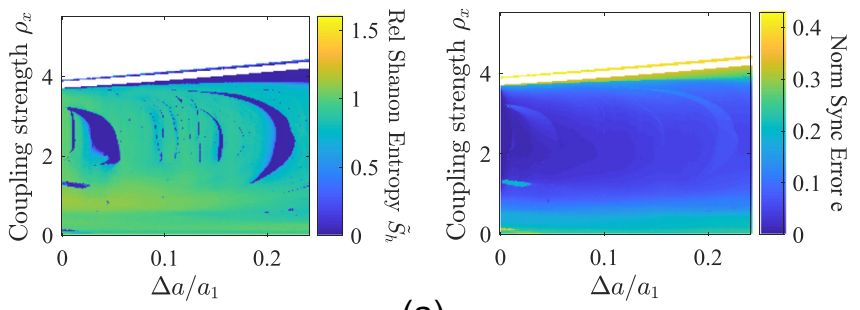

(a)
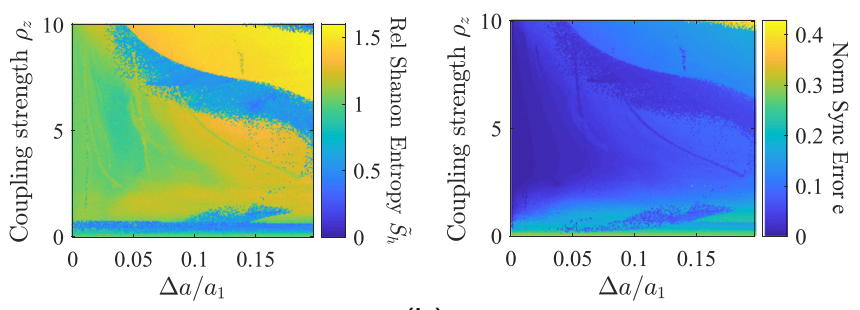

(b)
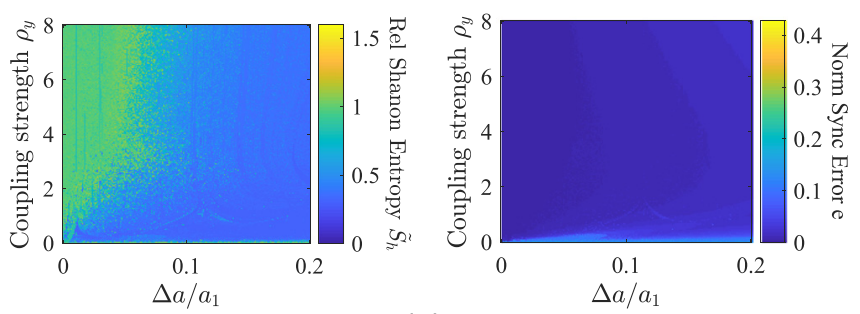

(c)

FIG. 11. Color maps of the relative Shannon entropy $\tilde{S}_{h}$ (left column) and normalized synchronization error $e$ (right column) versus the coupling parameter and the relative mismatch $\Delta a / a_{1}$ between oscillators. Parameter values are (a) Rössler systems, $a_{1}=0.520, \omega_{1}=5.0, b=2$ and $c=4$; (b) Lorenz 84 systems, $a_{1}=0.28, \omega_{1}=1, b=4, F=8$, and $G=1$; and (c) Sprott A systems, $a_{1}=2.0$, and $\omega_{1}=3.41$.

return map is comparable with the one that an isolated Rössler would have for an intermediate value of the $a$ parameter [in between the maps of Figs. 2(b) and 2(c)]. Indeed, the map of the collective dynamics has four monotonic branches but branch 3 is shortened in comparison with branch 3 that the first oscillator displays when uncoupled [Fig. 2(b)] and is more developed than in the map associated with the second oscillator [Fig. 2(c)]. On the other hand, when increasing the mismatch to $\frac{\Delta a}{a_{1}}=24 \%\left(a_{1}=0.520\right.$ and $\left.a_{2}=0.395\right)$, the topology of the attractor is still apparently similar to the structure of a common Rössler funnel attractor [Fig. 12(b)] but the first-return map presents some features that are no longer recognizable like the additional branch " 1 " (perhaps it is possible to find parameter values for reproducing it with a single Rössler system, but this would require a global modelling technique which is not the purpose of this paper). In a first approximation, this first-return map can still be considered as an intermediate case between the maps produced by the two oscillators when isolated [Figs. 2(b) and 2(d)]. Compared with the map for $a_{1}=0.520$, branch 3 is removed and branch 2 is only slightly developed; compared with the map for $a_{2}=0.395$, branch 0 is more developed and branch 2 is already present. This is confirmed by the relative Shannon entropies, $\quad \tilde{S}_{2}=0.48<\tilde{S}_{1-2}=0.85<\tilde{S}_{1}=1.00$, where $\tilde{S}_{1}, \tilde{S}_{2}$, and $\tilde{S}_{1-2}$ are the relative entropies of the first, the second, and the two coupled Rössler systems, respectively. 


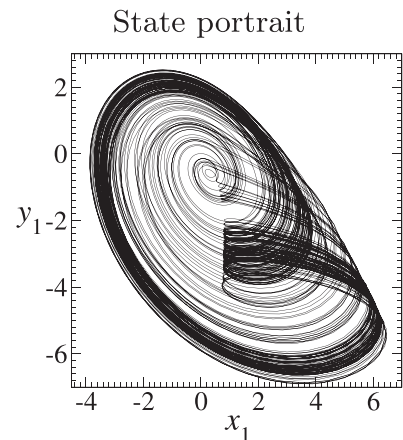

First-return map

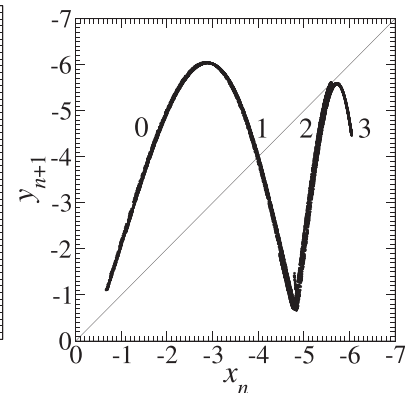

(a)
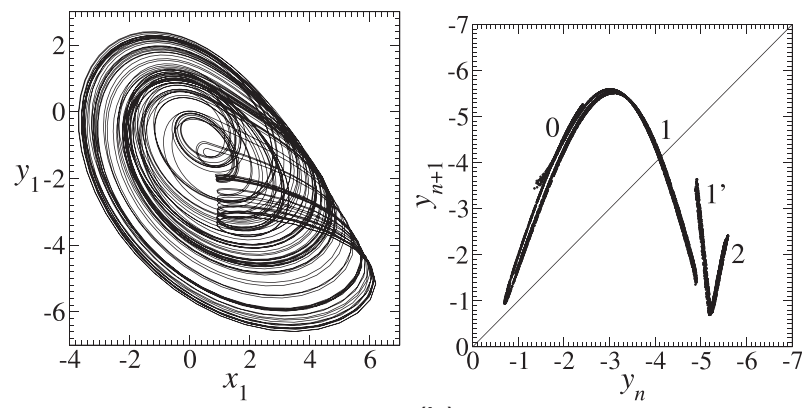

(b)

FIG. 12. Chaotic attractors produced by two Rössler systems coupled through the $x$ variable $\left(\rho_{x}=2.5\right)$ for a pair of mismatches in their $a$-values, $\Delta a / a_{1}=0.054$ and 0.24 , respectively [(a) $a_{2}=0.492, e=0.02$, and $\tilde{S}_{h}=1$ and (b) $a_{2}=0.395, e=0.06$, and $\left.\tilde{S}_{h}=0.85\right]$. In both cases, $a_{1}=0.520$, and the rest of parameters as in Fig. 11(a).

For the weakly dissipative case, there is a kind of Arnold tongue (green domain in Fig. 11(b)) extending over the region $2.0<\rho_{z}<10$, up to $\frac{\Delta a}{a_{1}} \approx 0.1$ for $\rho_{z} \approx 3.0$. In this region, the two oscillators are almost synchronous and the observed dynamics is very close to the reference one $\left(\tilde{S}_{h} \sim 1\right)$. Periodic windows are also present $\left(\tilde{S}_{h} \sim 0\right.$, blue strips) like the period-4 orbit shown in Fig. 13(a) for $\Delta a / a_{1}=0.11$ and $\rho_{z}=6.5$. On the other hand, yellow regions $\left(\tilde{S}_{h}>1\right)$ correspond to collective chaotic dynamics more developed than the isolated ones, as shown in Figs. 13(b) and 13(c). For instance, taking the isoline $\rho_{z}=6.5$ and moving to increasing values of the parameter mismatch, the resulting attractor still resembles the one produced by the reference oscillator, while the parameter mismatch is within the Arnold tongue [Fig. 13(b)]. For larger values of the parameter mismatch, the structure of the attractor is certainly different [Fig. 13(c)]: the layered structure of its Poincaré section is clearly blurred-as if it was noise contaminatedby the lack of synchronization and the Shannon entropy $S_{h}$ is increased by $50 \%$.

We argue that the low dissipation rate is the cause giving rise to a collective dynamics with a Shannon entropy greater than that observed separately. Such behavior is not observed in the strong dissipation regime, despite in the two cases, the visited domains by the attractors are of the same order. Hence, the multistability exhibited by the weakly dissipative systems is not explaining the way the synchronization error is processed but by the dissipation rate.

Finally, the conservative regime displays a much simpler case scenario. In most of the parameter space, the two

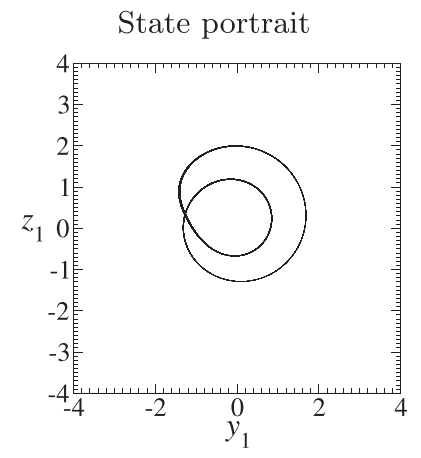

Poincaré section

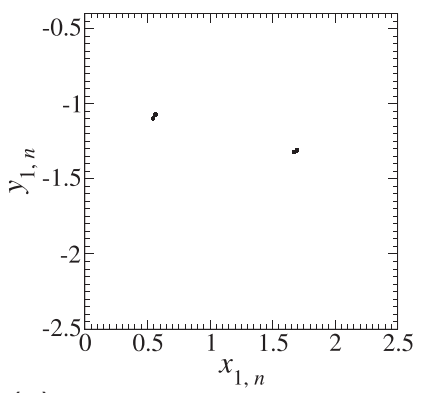

(a)
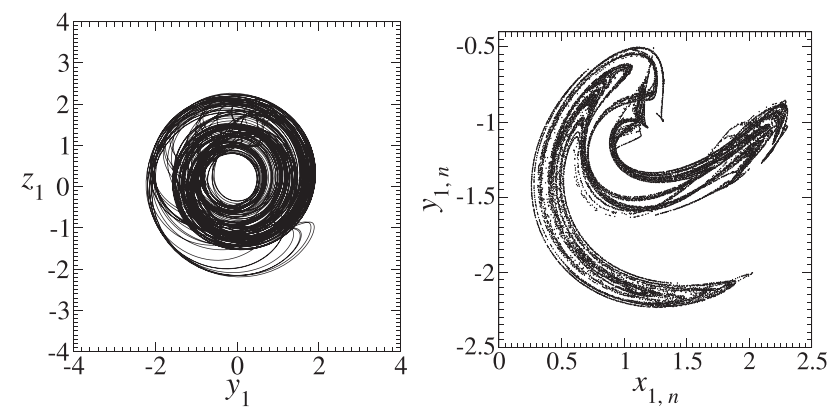

(b)
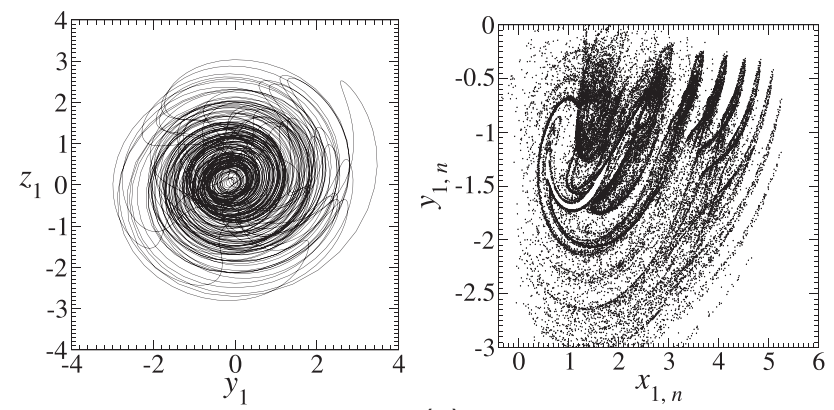

(c)

FIG. 13. Chaotic attractors produced by two nonidentical Lorenz 84 systems coupled through the variable $z\left(\rho_{z}=6.5\right)$ for three different values [(a) $a_{2}=0.250, e=0.02$, and $\tilde{S}_{h}=0.00 ;$ (b) $a_{2}=0.27, e=0.02$, and $\tilde{S}_{h}=0.99 ;$ (c) $a_{2}=0.225, e=0.07$, and $\left.\tilde{S}_{h}=1.49\right]$ of the parameter $a_{2}$ (mismatches $\Delta a / a_{1}=0.11,0.036$, and 0.2 ). In all cases, $a_{1}=0.28$, and the rest of parameters as in Fig. 11(b).

coupled systems are synchronous and the collective dynamics is settled either in the chaotic sea for small $\Delta a / a_{1}$ $\left[\tilde{S}_{h} \sim 1\right.$, green area in left panel of Fig. 11(c)] or performing a period-4 orbit for large $\Delta a / a_{1}\left[\tilde{S}_{h} \sim 0.3\right.$, blue area in left panel of Fig. 11(c)]. This means that the two coupled Sprott A oscillators cannot develop any other dynamics than those performed by the isolated systems and the selected one depends on the coupling and parameter mismatch. The period-4 orbit is actually a limit cycle, since there is a transient regime during which the synchronization error vanishes. Note that this limit cycle is clearly associated with the four islands observed in the two Poincare sections-shown in Figs. 8(b) and 8(c)—clearly structuring the chaotic sea (also shown in Fig. 9, black). Contrary to what is observed in the presence of dissipation, the conservative systems (i) are unable to remain chaotic when the parameter mismatch is large $\left(\frac{\Delta \omega}{\omega_{1}}>0.25\right)$ and (ii) are always synchronous. 

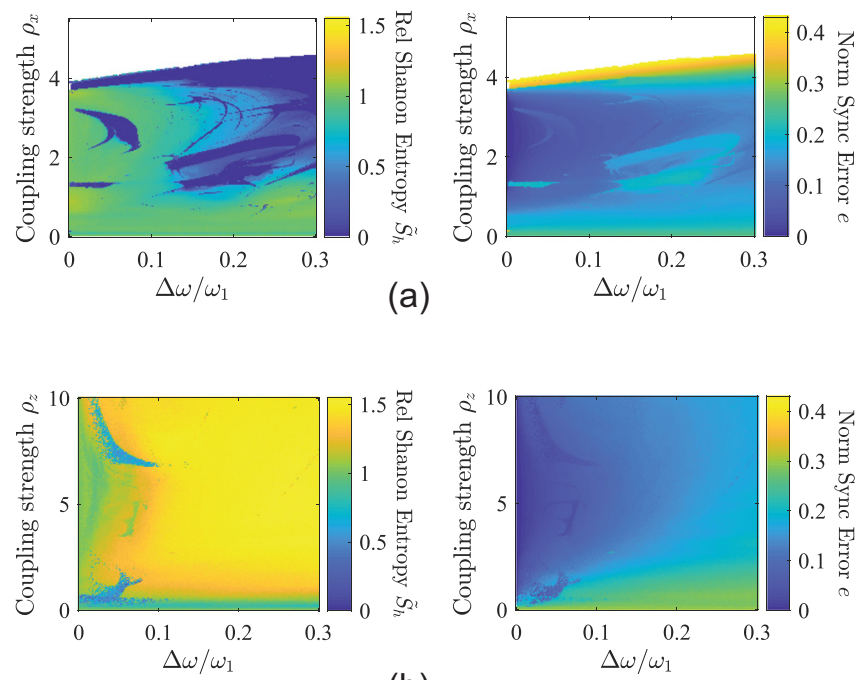

(b)
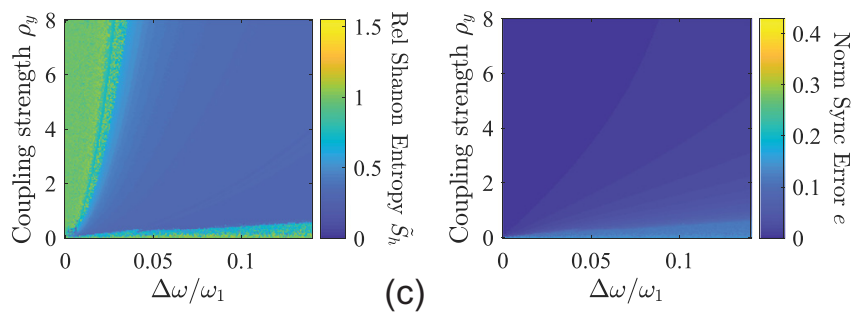

FIG. 14. Color maps of the relative Shannon entropy $\tilde{S}_{h}$ (left column) and normalized synchronization error $e$ (right column) versus the coupling parameter and the frequency mismatch $\Delta \omega / \omega_{1}$. Parameter values are (a) Rössler systems, $a_{1}=a_{2}=0.520, \omega_{1}=5.0$, and the rest of parameters as in Fig. 11(a); (b) Lorenz 84 systems, $a_{1}=a_{2}=0.28, \omega_{1}=1$, and the rest of parameters as in Fig. 11(b); (c) Sprott A systems, $a_{1}=a_{2}=2.0$ and $\omega_{1}=3.41$.

\section{B. Mismatch in the main frequency}

We will focus our attention only on the new features specially arising due to a frequency mismatch. Indeed, quite similar results are obtained when the mismatch $\frac{\Delta a}{a_{1}}$ is replaced by a frequency mismatch $\frac{\Delta \omega}{\omega_{1}}=\frac{\omega_{2}}{\omega_{1}}-1$ (Fig. 14), indicating that the frequency mismatch also plays the role of a bifurcation parameter. This means that the features we observe are mostly due to the error between the two oscillators and how it is released and not due to the nature of the dynamics producing that error. This is supported by the fact that the dynamics is topologically invariant under a frequency mismatch.

For the Rössler system, the collective dynamics is strongly reduced for $\frac{\Delta \omega}{\omega_{1}}>0.1$ as confirmed by the small relative Shannon entropy $\tilde{S}_{h}$ [Fig. 14(a)]. In particular, for $\frac{\Delta \omega}{\omega_{1}} \approx 0.3$, the attractor dynamics [see Fig. 15(a)] with such a first-return map cannot be retrieved from a single Rössler system. The double structure organized around the diagonal results from a banded attractor (the trajectory evolves on a band which crosses twice the Poincaré section before returning to itself) and the collective dynamics cannot be properly described in a three-dimensional space but actually in a sixdimensional space.

As for the weakly dissipative systems, a color map with also a very similar structure as the one observed in Fig. 11(b) has been obtained for $\frac{\Delta \omega}{\omega_{1}}<0.1$ [note, for instance, the blue strip observed in the upper part of Fig. 14(b)]. For $\frac{\Delta \omega}{\omega_{1}}>0.1$,
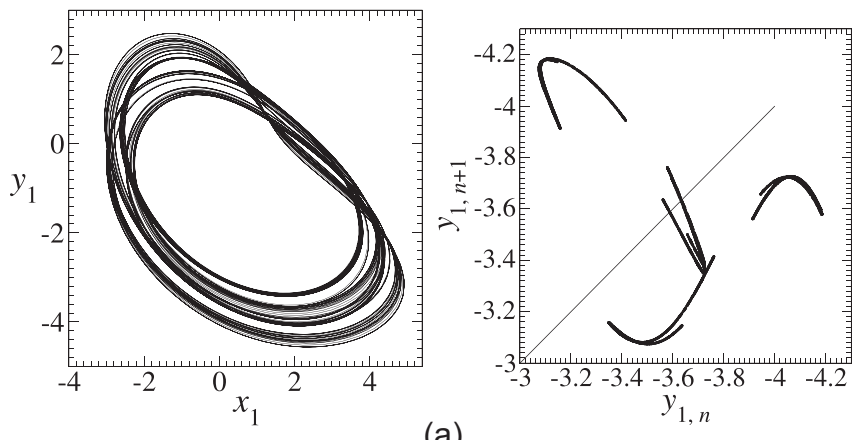

(a)
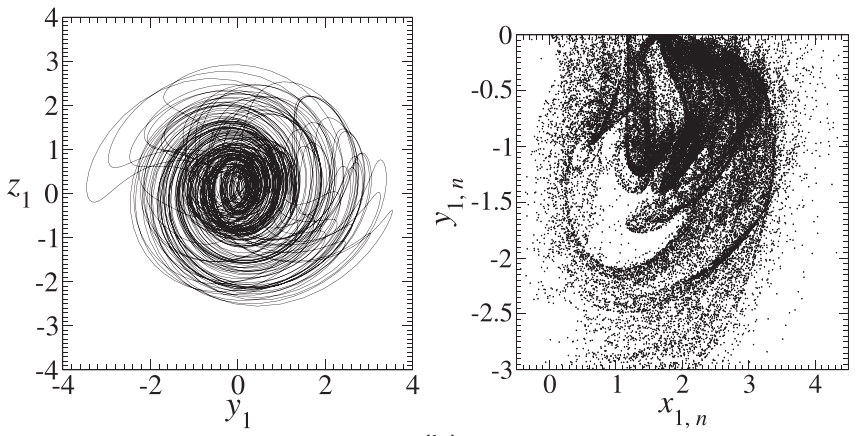

(b)

FIG. 15. Chaotic attractors (left column) produced by two coupled (a) Rössler oscillators: $\omega_{2}=6.5, e=0.14$, and $S_{h}=0.41$ (with its first-return map to the right) and (b) Lorenz 84: $\omega_{2}=1.3, e=0.10$, and $S_{h}=2.20$ (with its Poincaré section to the right) with a relative frequency mismatch $\Delta \omega / \omega_{1}=0.3$. Coupling parameter values are $\rho_{x}=2.5$ in (a) and $\rho_{z}=6.5$ in (b), respectively. Other parameter values are same as in Fig. 14.

the collective dynamics is nearly always associated with a relative Shannon entropy greater than 1.5. As an illustrative example, the resulting attractor for $\frac{\Delta \omega}{\omega_{1}}=0.3$ is shown in Fig. 14(b). It has a shape roughly similar to the one discussed in Fig. 13, with its Poincaré section no longer presenting a clear structure and not being distinguishable from a strongly noise-contaminated one. The synchronization error is not dissipated, and the resulting dynamics cannot be viewed as produced by a single isolated oscillator.

In the case of the Sprott A system, we recover the two types of dynamics we observed with a parameter mismatch, either a chaotic sea rather similar to the original one for $\frac{\Delta \omega}{\omega_{1}} \leqq 0.01$ or the period-4 orbit otherwise. There is a tiny region for small coupling values where it is possible to have a chaotic trajectory even for large frequency mismatch. Therefore, for the conservative case, it is difficult to get a chaotic dynamics when the systems are no longer identical.

The dependence on the initial conditions was very weak and does not affect the results in a significant way for any of the three types of systems. This is not surprising at all for the Rössler system because multistability is quite rare observed in the bifurcation diagram. On the contrary, the weakly dissipative Lorenz 84 system typically exhibits multistability. Nevertheless, such a lack of dependence to initial conditions may be explained by the fact that the different displayed attractors are always in the same domain of the state space. In the case of the conservative system, as long as the initial conditions are chosen within the chaotic sea, we obtained similar results. 


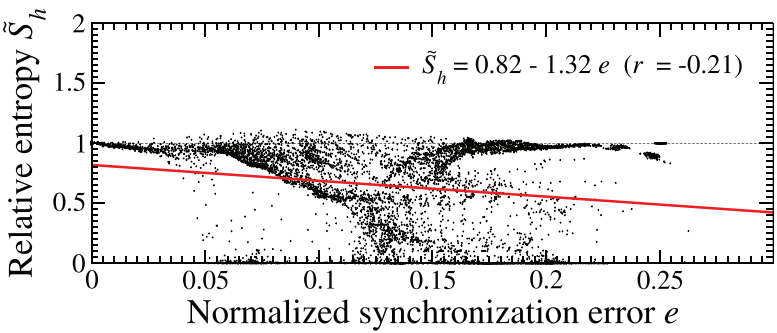

(a)

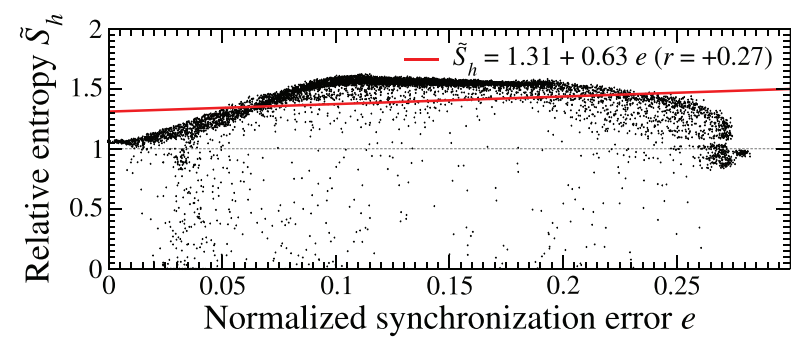

(b)

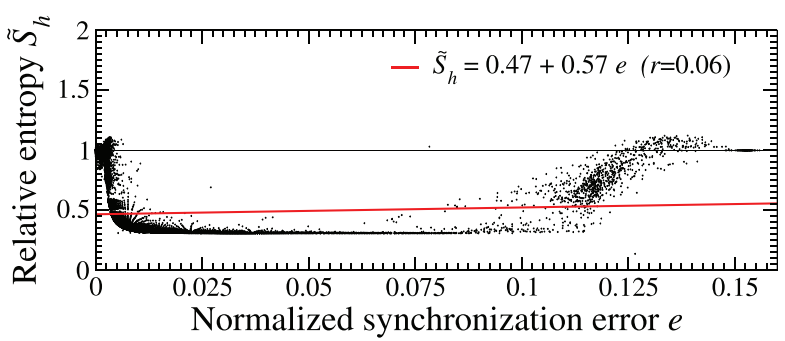

(c)

FIG. 16. Relative Shannon entropy $\tilde{S}_{h}$ versus the normalized synchronization error $e$ for (a) coupled Rössler, (b) Lorenz 84, and (c) Sprott A systems. Each dot corresponds to a particular value of the frequency mismatch and coupling strength within the same range as in Fig. 14 and $a_{1}=a_{2}$. The red straight lines are linear fittings.

In order to condense in a single picture the different behaviors observed as a function of the dissipation rate, we report in Fig. 16 the relative Shannon entropy $\tilde{S}_{h}$ as a function of the normalized synchronization error $e$, where each dot corresponds to a given pair of coupling strength and frequency mismatch values. In the three considered scenarios, strong, weak, and zero dissipation, points with $\tilde{S}_{h} \simeq 1$ occurring for small and large values of the synchronization error, correspond to situations of small frequency mismatch and small coupling strength, respectively. Outside this region, we observe three completely different trends. For the strongly dissipative system, the $\tilde{S}_{h}$ almost always remains below the reference value $\left(\tilde{S}_{h}<1\right)$ independently of the synchronization error. This implies that a strong dissipation induces a reduction of the complexity of the collective dynamics: as we observed with a parameter mismatch the resulting dynamics is either intermediate between the dynamics of the two isolated coupled oscillators or completely different from the original dynamics but with an attractor structured around a small population of periodic orbits, as indicated by the small relative Shannon entropy. For the weak dissipative systems, the synchronization error between the two coupled systems cannot be released. Since the trajectory does not converge quickly on the manifold of the original attractor, the resulting dynamics is more developed than the original one $\left(\tilde{S}_{h}>1\right)$. For conservative systems, the relative Shannon entropy is either close to 1 or quite small $(<0.5)$ : the collective dynamics is either in a chaotic sea very similar to the original one or in a period-4 orbit. It should be noted that the Shannon entropy remains close to 1 as long as the synchronization error is below $0.03(e<0.01)$ for strong (weak) dissipation and $e<0.005$ for the conservative system. This suggests that the higher the dissipation, the higher the synchronization error that the system can handle without changing the attractor or, equivalently, without changing the relative Shannon entropy.

\section{CONCLUSION}

Synchronization is an important dynamical process occurring in networked complex systems. While it has been widely reported in the case of strong dissipation, it was almost never investigated in weakly dissipative or conservative systems. Moreover, it has been mainly considered in nearly identical coupled units. We investigated the dynamical path followed by coupled systems whose parameter or frequency mismatch can be significant. We carried out such a study for strongly, weakly, and conservative systems to enlighten the possible differences arising during the synchronization process.

On the one hand, when the coupled systems are identical or slightly different, we choose a coupling form ensuring full synchronization. In those cases, the observed behavior does not depend on the dissipation rate and the synchronization manifold is equivalent to the dynamics produced by an isolated system. On the other hand, when the parameter mismatch is sufficiently large for producing a trajectory that visits different domains of the state space, the resulting dynamics strongly depends on the dissipation rate. For strongly dissipative systems, as the Rössler example, the collective dynamics is in between the original attractors. In the case of weakly dissipative systems as the Lorenz 84 systems, the dynamics is found to be most often more developed than the dynamics of the isolated ones. Our results suggest that this is mainly due to the low dissipation rate which does not allow the fast release of the synchronization error. The case of conservative systems as the Sprott A system is easier to interpret because the dynamics is either the chaotic sea exhibited by the isolated system or a periodic orbit when the parameter mismatch is too large. In the Sprott A system, the orbit is always a period- 4 orbit around which the chaotic sea is organized. Note that we might speak of a limit cycle, although the oscillator is conservative, because there is a transient regime along which the synchronization error is progressively reduced to a very small value.

When there exists a frequency mismatch instead, the whole previous scenario described for a parameter mismatch is replicated as long as the mismatch is not too large. For large frequency mismatch, strongly dissipative systems reveal high dimensional new attractors, while weakly dissipative systems organize into strongly noise contaminated attractors, only approximately resembling the original attractor produced by an isolated system. Coupled conservative systems either produce a chaotic sea similar to the original 
one or a period-4 orbit. Consequently, the dissipation rate strongly affects the way the coupled systems react to a (large) frequency mismatch.

Finally, despite we used three different systems for investigating the influence of the dissipation rate on synchronization, we explored the possibility of having a single system in which the dissipation rate could progressively be changed by tuning one of its parameters as in the modified Sprott A system proposed by Cang and coworkers. ${ }^{37}$ However, it was impossible to produce a chaotic attractor with a small dissipation rate (only limit cycles were obtained), and chaotic behavior was only observed for the conservative case (thus equivalent to the Sprott A system) or for strong dissipation (thus producing a Lorenz-like attractor). Investigating the effect of the dissipation rate by just using the same system remains, therefore, a subject of a great interest.

${ }^{1}$ O. E. Rössler, “An equation for continuous chaos," Phys. Lett. A 57(5), 397-398 (1976).

${ }^{2}$ R. Gilmore and M. Lefranc, The Topology of Chaos (Wiley, 2002).

${ }^{3}$ C. Letellier, E. Roulin, and O. E. Rössler, "Inequivalent topologies of chaos in simple equations," Chaos, Solitons Fractals 28, 337-360 (2006).

${ }^{4}$ M. Rosalie and C. Letellier, "Systematic template extraction from chaotic attractors: I. Genus-one attractors with an inversion symmetry," J. Phys. A 46, 375101 (2013).

${ }^{5}$ J. Plumecoq and M. Lefranc, "From template analysis to generating partitions I: Periodic orbits, knots and symbolic encodings," Physica D 144, 231-258 (2000).

${ }^{6} \mathrm{H}$. Poincaré, Les Méthodes Nouvelles de la Mécanique Céleste (GauthierVilard, Paris, 1899), Vol. 3.

${ }^{7}$ P. Cvitanović, "Periodic orbits as the skeleton of classical and quantum chaos," Physica D 51, 138-151 (1991).

${ }^{8} \mathrm{G}$. B. Mindlin and R. Gilmore, "Topological analysis and synthesis of chaotic time series," Physica D 58, 229-242 (1992).

${ }^{9}$ A. A. King, W. M. Schaffer, C. Gordon, J. Treat, and M. Kot, "Weakly dissipative predator-prey systems," Bull. Math. Biol. 58(5), 835-859 (1996).

${ }^{10} \mathrm{P}$. Glendinning, "Inaccessible attractors of weakly dissipative systems," Nonlinearity 10(2), 507-522 (1997).

${ }^{11}$ A. Celletti, "Weakly dissipative systems in celestial mechanics," Lect. Notes Phys. 729, 67-90 (2007).

${ }^{12}$ D. S. Almeida Júnior, M. L. Santos, and J. E. Muñoz Rivera, "Stability to weakly dissipative Timoshenko systems," Math. Methods Appl. Sci. 36(14), 1965-1976 (2013).

${ }^{13}$ R. F. Nagaev, "General problem of synchronization in an almost conservative system," J. Appl. Math. Mech. 29(5), 801-809 (1965).

${ }^{14}$ L. Zonghua and C. Shigang, "Synchronization of a conservative map," Phys. Rev. E 56(2), 1585-1589 (1997).

${ }^{15}$ S. Vaidyanathan and S. Pakiriswam, "A novel conservative chaotic system and its generalized projective synchronization via adaptive control," J. Eng. Sci. Technol. Rev. 8(2), 52-60 (2015).

${ }^{16}$ O. I. Olusola, U. E. Vincent, and A. N. Njah, "Synchronization, multistability and basin crisis in coupled pendula," J. Sound Vib. 329, 443-456 (2010).
${ }^{17}$ A. N. Pisarchik and U. Feudel, "Control of multistability," Phys. Rep. 540, $167-218$ (2014).

${ }^{18}$ H. B. Lin, Elementary Symbolic Dynamics and Chaos in Dissipative Systems (World Scientific Publishing, 1989).

${ }^{19} \mathrm{C}$. Letellier, P. Dutertre, and B. Maheu, "Unstable periodic orbits and templates of the Rössler system: Toward a systematic topological characterization," Chaos 5(1), 271-282 (1995).

${ }^{20}$ S. Mangiarotti, M. Peyre, and M. Huc, "A chaotic model for the epidemic of Ebola virus disease in West Africa (2013-2016)," Chaos 26(11), 113112 (2016).

${ }^{21}$ C. Letellier, "Estimating the Shannon entropy: Recurrence plots versus symbolic dynamics," Phys. Rev. Lett. 96, 254102 (2006).

${ }^{22}$ L. L. Trulla, A. Giuliani, J. P. Zbilut, and C. L. Webber, Jr., "Recurrence quantification analysis of the logistic equation with transients," Phys. Lett. A 223, 255-260 (1996).

${ }^{23}$ R. Naeck, D. Bounoiare, U. S. Freitas, H. Rabarimanantsoa, A. Portmann, F. Portier, A. Cuvelier, J.-F. Muir, and C. Letellier, "Dynamics underlying patient-ventilator interactions during nocturnal noninvasive ventilation," Int. J. Bifurcation Chaos 22(2), 1250030 (2012).

${ }^{24}$ E. N. Lorenz, "Irregularity: A fundamental property of the atmosphere," Tellus A 36, 98-110 (1984).

${ }^{25}$ A. Shil'nikov, G. Nicolis, and C. Nicolis, "Bifurcation and predictability analysis of a low-order atmospheric circulation model," Int. J. Bifurcation Chaos 5(6), 1701-1711 (1995).

${ }^{26}$ J. G. Freire, C. Bonatto, C. C. DaCamara, and J. A. Gallas, "Multistability, phase diagrams, and intransitivity in the Lorenz-84 low-order atmospheric circulation model," Chaos 18(3), 033121 (2008).

${ }^{27}$ L. van Veen, "Baroclinic flow and the Lorenz-84 model," Int. J. Bifurcation Chaos 13(8), 2117-2139 (2003).

${ }^{28} \mathrm{H}$. Wang, Y. Yu, and G. Wen, "Dynamical analysis of the Lorenz-84 atmospheric circulation model," J. Appl. Math. 2014, 296279.

${ }^{29}$ J. C. Sprott, "Some simple chaotic flows," Phys. Rev. E 50(2), 647-650 (1994).

${ }^{30}$ L. M. Pecora and T. L. Carroll, "Synchronization in chaotic systems," Phys. Rev. Lett. 64(8), 821-824 (1990).

${ }^{31}$ S. Boccaletti, J. Kurths, G. Osipov, D. L. Valladares, and C. S. Zhou, "The synchronization of chaotic systems," Phys. Rep. 366(1-2), 1-101 (2002).

${ }^{32}$ L. M. Pecora and T. L. Carroll, "Master stability functions for synchronized coupled systems," Phys. Rev. Lett. 80(10), 2109-2112 (1998).

${ }^{33}$ S. Boccaletti, V. Latora, Y. Moreno, M. Chavez, and D.-U. Hwang, "Complex networks: Structure and dynamics," Phys. Rep. 424, 175-308 (2006).

${ }^{34}$ I. Sendiña-Nadal, S. Boccaletti, and C. Letellier, "Observability coefficients for predicting the class of synchronizability from the algebraic structure of the local oscillators," Phys. Rev. E 94, 042205 (2016).

${ }^{35}$ A. Pikovsky, M. Rosenblum, and J. Kurths, Synchronization: A Universal Concept in Nonlinear Sciences (Cambridge University Press, 2003).

${ }^{36}$ S. Boccaletti, A. A. Koronovsky, D. I. Trubetskov, A. E. Khramov, and A. E. Khramova, "Stability of the synchronous state of an arbitrary network of coupled elements," Radiophys. Quantum Electron. 49(10), 826-833 (2006).

${ }^{37}$ S. Cang, A. Wu, Z. Wang, and Z. Chen, "On a 3-D generalized Hamiltonian model with conservative and dissipative chaotic flows," Chaos, Solitons Fractals 99, 45-51 (2017).

${ }^{38}$ The time averaged trace is calculated over 80000 points taken along a trajectory in the chaotic attractor.

${ }^{39}$ The visited volume $\mathcal{V}$ is approximated by counting the pixels visited by 80000 intersections with the Poincare section $\left(400 \times 400\right.$ pixels $\left.^{2}\right)$. 